\title{
Nonparametric Prediction Intervals of generalized order statistics from two independent sequences
}

\author{
M. M. Mohie El-Din ${ }^{1}$ and W. S. Emam ${ }^{2 *}$ \\ ${ }^{1}$ Department of Mathematics, Faculty of Science, Al-Azhar University, Nasr City, Cairo, Egypt \\ ${ }^{2}$ Department of Basic Science, Faculty of Engineering, British University in Egypt, Al-Shorouq City, Cairo, Egypt
}

\begin{abstract}
This paper, discusses the problem of predicting future a generalized order statistic of an iid sequence sample was drawn from an arbitrary unknown distribution, based on observed also generalized order statistics from the same population. The coverage probabilities of these prediction intervals are exact and free of the parent distribution $F()$. Prediction formulas of ordinary order statistics and upper record values are extracted as special cases from the productive results. Finally, numerical computations on several models of ordered random variables are given to illustrate the proposed procedures.
\end{abstract}

Keywords: Prediction intervals; Generalized order statistics; Coverage probability; Confidence intervals; Two-sample prediction.

\section{Introduction}

The prediction subject of unobserved data has received a considerable attention in the literatures during the last two decades. Several applications of the prediction problems can be found in medical, engineering, stock market studies. Prediction subject have been categorized generally into two types: one-sample prediction and two-sample prediction. One-sample prediction case is based on a sequence of observations, experimenter seeks to predict the future random variables from the same sequence. In two-sample prediction type, using the available observations of past information sample, experimenter interested in (with some level of confidence) predicting some statistics in a future unobserved sample from the same underlying distribution $F()$. The past sample and the future sample are iid. Also, the predictor can be either point or interval. Such an interval is said to be a prediction interval (PIs) for the statistic of interest. Also, the prediction interval can be parametric (if it depends on the distribution parameters) or nonparametric (distribution-free). Often the observed data may not appropriate with certain distribution. Therefore, the results may include some error resulting from the mistakes in determining the suitable distribution. Distribution-free predictive inference is a statistical procedure to learn from data in the absence of prior knowledge and using only few modeling assumptions. The proposed prediction intervals are distribution-free, i.e. the corresponding coverage probabilities are known exactly without any assumption about the parent distribution other than being continuous. An exact expression for the prediction coefficient of these intervals is derived.

Many contexts have taken place in the distribution-free PIs direction using several assumptions [1]-[10]. But all these articles shared in one to one prediction way, i.e. predict a future certain type of samples based on another one. For the purpose of generalization, Mohie El-Din and Emam [11] discussed the predicting of future generalized order statistics, as well as outer and inner PIs based on ordinary order statistics. For more generalization, this article discusses the predicting of future generalized order statistics based on generalized order statistics. The study was conducted over all assumptions of generalized order statistics ( $g O S s)$ [12] and [13] Paper is organized as follows: In section 2, some preliminaries are given. In Section 3, distribution-free PIs for a gOS from a future Y-sequence of iid random variables, based on also $\mathrm{gOSs}$ from the $\mathrm{X}$-sequence are derived. Section 4, includes numerical computations. Finally, conclusions are given in section 5.

This paper aims to construct nonparametric PIs for future $\mathrm{gOSs}$ based on $g O S s$, therefore, all the previous researches in the field of nonparametric PIs for future Y-sample based on another Xsample (which can be obtained as a special model of gOSs as table 1 considerations) is a special case of this paper. Some numerical results of $o O S s$ and Krecord (for some $K \in N$ ) as special models of gOSs case I; and oOSs, nonI, Seque, Pfief, PCOs with two stages $\left(m_{r}=0\right.$ if $r \neq r_{1}, m_{r_{1}}=n_{1}, k=v-n_{1}-n+1$, $\gamma_{r}=v-n_{1}-r+1$ if $r \leq r_{1}, \gamma_{r}=v-n_{1}-r+1$ if $r>r_{1}$, see, [12] p.48), Trunc and $k_{n}$ rec as special models of case II.

\section{Preliminaries}

The concept of gOSs was introduced by Kamps [12] and was developed by Kamps and Cramer [13]. This concept was introduced as a unified approach to several models of ordered random variables such as, ordinary order statistics $(o O S s)$, order statistics with non-integral sample size (nonI), K-records model (Krecord), upper 
record values (record), sequential order statistics (Seque), Pfeifer's record model ( $P$ feif), progressively Type-II right-censored order statistics sample (PCOs), ordering via truncation (Trunc) and $k_{n}$-record values $\left(k_{n} r e c\right)$. Table 1 , shows these models as special cases of $g O S s$. Let $n \in N, \widetilde{m}=\left(m_{1}, \ldots, m_{n-1}\right) \in R_{n-1}$, if $n \geq 2$ ( $\widetilde{m} \in R$, arbitrary if $n=1) k \geq 1$, be given constants such that for all $1 \leq i \leq n-1, \gamma_{i}=k+n-i+M_{i}>0$, where $M_{i}=\sum_{j=i}^{n-1} m_{j}$. We may classify $\mathrm{gOSs}$ into the following two cases:

Case I: If $m_{1}=m_{2}=\ldots=m_{n-1}=m$, the probability density function (pdf) of $X_{r, n, m, k}$, as introduced by Kamps [12], can be written as

$$
f_{X_{r, n, m, k}}(x)=\frac{c_{r-1}}{(r-1) !} \bar{F}^{\gamma_{r}-1}(x) f(x) g_{m}^{r-1}(F(x)),
$$

where $c_{r-1}=\prod_{j=1}^{r} \gamma_{j}, \gamma_{j}=k+(n-j)(m+1)$, $g_{m}(z)=h_{m}(z)-h_{m}(0), 0<z<1$, such that

$$
h_{m}(z)= \begin{cases}\frac{-(1-z)^{m+1}}{m+1}, & m \neq-1, \\ -\ln (1-z), & m=-1 .\end{cases}
$$

The survival function of $X_{r, n, m, k}$, is given by:

$$
\bar{F}_{X_{i, n, m, k}}(x)=c_{i-1}(1-F(x))^{\gamma_{i}} \sum_{v=0}^{i-1} \frac{g_{m}^{v}(F(x))}{v ! c_{i-v-1}}, 1 \leq i \leq n .
$$

Case II: If $\gamma_{i} \neq \gamma_{j}, i, j=1,2, \ldots, n-1$ and $i \neq j$, the pdf of $X_{r, n, \widetilde{m}, k}$ which is given in Eq.(1) as be introduced by Kamps and Cramer [13], is given by:

$$
f_{X_{r, n, \tilde{m}, k}}(x)=c_{r-1} \sum_{i=1}^{r} a_{i}(r)(\bar{F}(x))^{\gamma_{i}-1} f(x),
$$

where $a_{i}(r)=\prod_{j=1, j \neq i}^{r} \frac{1}{\gamma_{j}-\gamma_{i}}, 1 \leq r \leq n$ and $\gamma_{i}=k+n-i+M_{i}>0$ And the survival function of $X_{r, n, \widetilde{m}, k}$, is given by:

$$
\bar{F}_{X_{i, n, \tilde{n}, k}}(x)=c_{i-1} \sum_{v=1}^{i} \frac{a_{v}(i)}{\gamma_{v}}(1-F(x))^{\gamma_{v}} .
$$

Let $c_{i-1}, m, a_{i}(r)$ and $\gamma_{i}$ denote the past scheme $\mathrm{X}$ constants, and $c_{r-1}^{*}, m^{*}, a_{i}^{*}(r)$ and $\gamma_{r}^{*}$ follow the future unobserved scheme $\mathrm{Y}$. The prediction coefficients $\phi$ not only depend on subscripts, but also depend on observed sample sizes $n$ and $n^{*}$ in addition the constants $k$ and $k^{*}$, i.e. $\phi()=.\phi\left(., n, k ; ., n^{*}, k^{*}\right)$.

Table 1: Some special cases of $g O S s$, with $\lambda_{i}, \alpha_{i} \in \mathbf{R}^{+}$,

\begin{tabular}{lccc}
\multicolumn{4}{c}{$1 \leq i \leq n-1}$. \\
\hline Model & $m_{r}$ & $k$ & $\gamma_{r}$ \\
\hline oOSs & 0 & 1 & $n-r+1$ \\
nonI & 0 & $\alpha-n+1$ & $\alpha-r+1$ \\
Krecord & -1 & $k \in \mathscr{N}$ & $k$ \\
record & -1 & 1 & 1 \\
Seque & $(n-r+1) \alpha_{r}-$ & $\alpha_{n}$ & $(n-r+1) \alpha_{r}$ \\
& $(n-r) \alpha_{r+1}-1$ & & \\
Pfeif & $\lambda_{r}-\lambda_{r+1}-1$ & $\lambda_{n}$ & $\lambda_{r}$ \\
PCOs & $R_{r} \in \mathscr{N}_{0}$ & $R_{m}+1$ & $m-r+1+M_{i}$ \\
Trunc & $\alpha_{r} k_{r}-\alpha_{r+1} k_{r+1}-1$ & $\alpha_{n} k_{n}$ & $\alpha_{r} k_{r}$ \\
$k_{n}$ rec & $\lambda_{r} k_{r}-\lambda_{r+1} k_{r+1}-1$ & $\lambda_{n} k_{n}$ & $\lambda_{r} k_{r}$ \\
\hline
\end{tabular}

Lemma1. Based on X-sample observations, suppose we are interested in obtaining $(1-\alpha) 100 \%$ distribution-free upper pound PIs for $Y_{r}$ from a future Y-sample of the form $\left(-\infty, X_{i}\right), 0 \leq \alpha \leq 1$, such that, the coverage probability $P\left(X_{i} \geq Y_{r}\right)=1-\alpha$. We refer to the interval $\left(-\infty, X_{i}\right)$ as $(1-\alpha) 100 \%$ PI for $Y_{r}$. Upon the non-parametric prediction procedure by assuming that $Y_{r}$ is continuous random variable. Then, we get

$$
\phi(i ; r)=P\left(X_{i} \geq Y_{r}\right)=\int_{-\infty}^{\infty} P\left(X_{i} \geq y\right) f_{Y_{r}}(y) d y .
$$

Distribution-free prediction attempt transforming integration base from the random variable to the survival function, to distribute $\bar{F}()$ randomly as standard uniform $(0,1)$ random variable. Therefore, the sampling distribution doesn't appear in final results. The coverage probabilities of these PIs intervals are exact and are free of the parent distribution $F()$, and depend only on the prefixed subscripts of the samples. Therefor, $\phi(i ; r)=1-\alpha$ represents the prediction coefficient which does not depend on the parameters of the parent distribution $F$, it depends only on the random variable's positions (the indices $i$ and $r$ ) in addition modeling prefixes. Here, $X_{i}$ are the upper bounds of the prediction interval for $Y_{r}$. Under the assumptions of lemma1, assume we are interested in obtaining $(1-\alpha) 100 \%$ distribution-free two sided PIs for $Y_{r}$ from a future Y-sample of the form $\left(X_{i}, X_{j}\right), 1 \leq i<j$, such that, the coverage probability $P\left(X_{i} \leq Y_{r} \leq X_{j}\right)=1-\alpha$. We refer to the interval $\left(X_{i}, X_{j}\right)$ as $(1-\alpha) 100 \%$ PI for $Y_{r}$. Then, we get

$$
\begin{aligned}
p\left(X_{i} \leq Y_{r} \leq X_{j}\right) & =P\left(X_{j} \geq Y_{r}\right)-P\left(X_{i} \geq Y_{r}\right) \\
& =\phi(j ; r)-\phi(i ; r) .
\end{aligned}
$$

Such that, $p\left(X_{i} \leq Y_{r} \leq X_{j}\right)=1-\alpha$ presents the coverage probability for $Y_{r}$. Thus, we have $\left(X_{i}, X_{j}\right)$ is a prediction interval for $Y_{r}$, Here, $X_{i}$ and $X_{j}$ are the lower and upper bounds of the prediction interval for $Y_{r}$, respectively.

\section{PIs for individual $g O S s$}

In this section, we obtain one and two-sided distribution-free PIs for a future $r^{t h} \operatorname{gOSs} Y_{r, n^{*}, \widetilde{m^{*}}, k^{*}}, 1 \leq i \leq n^{*}$ based on the endpoints of observed $g O S s$. Let $Y_{1, n^{*}, \widetilde{m^{*}}, k^{*}}, \ldots, Y_{n^{*}, n^{*}, \widetilde{m^{*}, k^{*}}}$ be $n^{*} g O S s$ based on the continuous cumulative distribution function (cdf) $F$ with density function (pdf) $f$ from a future $Y$-sequence of i.i.d. random variables, under assumption $m_{1}^{*}=m_{2}^{*}=\ldots=m_{n-1}^{*}=m^{*}$, or $\gamma_{i}^{*} \neq \gamma_{j}^{*}, i, j=1,2, \ldots, n^{*}-1$ and $i \neq j$, and let $X_{l, n, \widetilde{m}, k}$ be $i^{\text {th }}$ gOSs from another observed random sample, under assumption $m_{1}=m_{2}=\ldots=m_{n-1}=m$, or $\gamma_{i} \neq \gamma_{j}, i, j=1,2, \ldots, n^{*}-1$ and $i \neq j$, and further let the underling distribution of the two samples be the same. We are interested here in obtaining one and two-sided distribution-free PIs for a future $r^{t h} \operatorname{gOSs} Y_{r, n^{*}, \widetilde{m^{*}, k^{*}}}, 1 \leq r \leq n^{*}$ based on the endpoints of observed $g O S s$. The coverage probabilities of this PIs are exact and do not depend on the sampling distribution.

Theorem 1 . Let $\left\{X_{i, n, m, k}, 1 \leq i \leq n\right\}$ under assumption $m_{1}=m_{2}=\ldots=m_{n-1}=m$, and $\left\{Y_{r, n^{*}, m^{*}, k^{*}}, 1 \leq r \leq n^{*}\right\}$ under assumption $m_{1}^{*}=m_{2}^{*}=\ldots=m_{n-1}^{*}=m^{*}$, be two independent $g O S s$ from continuous cdf $F$. then $\left(-\infty, X_{i, n, m, k}\right), 1 \leq i \leq n$, is an upper prediction bound for the future $Y_{r, n^{*}, m^{*}, k^{*}}$, with the corresponding prediction coefficient, being free of $F$, given by:

$$
\begin{aligned}
& \phi_{1}\left(i, m ; r, m^{*}\right)=\sum_{v=0}^{i-1} C_{v}(i ; r) \times \\
& \begin{cases}\sum_{\lambda=0}^{v} \frac{b_{\lambda}^{v}(m) B\left(r, \frac{\gamma_{i}+\gamma_{r}^{*}+\lambda(m+1)}{m^{*}+1}\right)}{\left(m^{*}+1\right)^{r}}, & m \neq-1 ; m^{*} \neq-1, \\
\sum_{\lambda=0}^{v} \frac{(r-1) ! b_{\lambda}^{v}(m)}{\left(\gamma_{i}+k^{*}+\lambda(m+1)\right)^{r}}, & m \neq-1 ; m^{*}=-1, \\
\sum_{\eta=0}^{r-1} \frac{v ! b_{\eta}^{r-1}\left(m^{*}\right)}{\left(k+\gamma_{r}^{*}+\eta\left(m^{*}+1\right)\right)^{v+1}}, & m=-1 ; m^{*} \neq-1, \\
\frac{(v+r-1) !}{\left(k+k^{*}\right)^{v+r}}, & m=-1 ; m^{*}=-1,\end{cases}
\end{aligned}
$$

where $C_{v}(i ; r)=\frac{c_{i-1} c_{r-1}^{*}}{(r-1) ! v ! c_{i-v-1}}, b_{\lambda}^{v}(m)=\frac{(-1)^{\lambda}\left(\begin{array}{l}v \\ \lambda\end{array}\right)}{(m+1)^{v}}$ and $B(.,$.$) is a$ beta constant. 
Table 2: Some values of $p\left(X_{i, 20, m, k} \leq Y_{r, n^{*}, m^{*}, k^{*}} \leq X_{j, 20, m, k}\right)$ for some $i, j$ and $r$.

\begin{tabular}{|c|c|c|c|c|c|c|c|c|c|}
\hline \multicolumn{4}{|l|}{ Sample } & \multicolumn{6}{|l|}{1} \\
\hline$X$ & $r$ & $i$ & $j$ & $Y_{r: 25}$ & record & 2 record & 3 record & 4record & 5 recorc \\
\hline \multirow{19}{*}{$X_{l: 20}$} & \multirow[t]{5}{*}{1} & \multirow[t]{3}{*}{1} & 6 & 0.4889 & 0.1923 & 0.3276 & 0.4228 & 0.4892 & 0.5351 \\
\hline & & & 12 & 0.5000 & 0.4231 & 0.6268 & 0.7219 & 0.7619 & 0.7732 \\
\hline & & & 18 & 0.5000 & 0.6538 & 0.8234 & 0.8562 & 0.8482 & 0.8278 \\
\hline & & \multirow[t]{2}{*}{3} & 6 & 0.1062 & 0.1154 & 0.18803 & 0.2320 & 0.2566 & 0.2682 \\
\hline & & & 18 & 0.1173 & 0.5769 & 0.6838 & 0.6654 & 0.6156 & 0.5609 \\
\hline & \multirow[t]{4}{*}{3} & \multirow[t]{3}{*}{1} & 6 & 0.7593 & 0.0037 & 0.0222 & 0.0574 & 0.1055 & 0.1617 \\
\hline & & & 12 & 0.8808 & 0.0306 & 0.1441 & 0.2983 & 0.4508 & 0.5812 \\
\hline & & & 18 & 0.8827 & 0.1315 & 0.4334 & 0.6783 & 0.8267 & 0.9065 \\
\hline & & 3 & 18 & 0.5000 & 0.1310 & 0.4298 & 0.6680 & 0.8060 & 0.8721 \\
\hline & \multirow[t]{5}{*}{6} & \multirow[t]{3}{*}{1} & 18 & 0.9886 & 0.0028 & 0.0503 & 0.1835 & 0.3661 & 0.5443 \\
\hline & & & 22 & 0.9889 & 0.0226 & 0.2249 & 0.5212 & 0.7460 & 0.8755 \\
\hline & & & 25 & 0.9889 & 0.2096 & 0.6889 & 0.9063 & 0.9734 & 0.9923 \\
\hline & & \multirow[t]{2}{*}{5} & 18 & 0.6371 & 0.0028 & 0.0503 & 0.1832 & 0.3647 & 0.5401 \\
\hline & & & 22 & 0.6374 & 0.0226 & 0.2248 & 0.5209 & 0.7447 & 0.8719 \\
\hline & \multirow[t]{5}{*}{10} & \multirow[t]{3}{*}{10} & 22 & 0.4998 & 0.0002 & 0.0167 & 0.1118 & 0.2950 & 0.5007 \\
\hline & & & 24 & 0.5000 & 0.0027 & 0.0948 & 0.3489 & 0.6156 & 0.7979 \\
\hline & & & 25 & 0.5000 & 0.0207 & 0.2692 & 0.6079 & 0.8240 & 0.9259 \\
\hline & & \multirow[t]{2}{*}{15} & 24 & 0.0782 & 0.0027 & 0.0947 & 0.3473 & 0.6063 & 0.7676 \\
\hline & & & 25 & 0.0782 & 0.0207 & 0.2691 & 0.6063 & 0.8146 & 0.8957 \\
\hline \multirow[t]{20}{*}{ record } & \multirow[t]{4}{*}{5} & \multirow[t]{3}{*}{1} & 4 & 0.1921 & 0.3320 & 0.6097 & 0.6489 & 0.6160 & 0.5674 \\
\hline & & & 8 & 0.1923 & 0.7749 & 0.8496 & 0.7599 & 0.6717 & 0.5980 \\
\hline & & & 20 & 0.1923 & 0.9680 & 0.8683 & 0.7627 & 0.6723 & 0.5981 \\
\hline & & 2 & 20 & 0.0235 & 0.8899 & 0.6488 & 0.4661 & 0.3446 & 0.2632 \\
\hline & \multirow[t]{6}{*}{10} & \multirow[t]{4}{*}{1} & 4 & 0.3820 & 0.0452 & 0.3051 & 0.5279 & 0.6400 & 0.6804 \\
\hline & & & 8 & 0.3846 & 0.3136 & 0.8108 & 0.9035 & 0.8817 & 0.8350 \\
\hline & & & 12 & 0.3846 & 0.6672 & 0.9615 & 0.9420 & 0.8924 & 0.8385 \\
\hline & & & 18 & 0.3846 & 0.9380 & 0.9823 & 0.9437 & 0.8926 & 0.8385 \\
\hline & & 2 & 18 & 0.0931 & 0.9331 & 0.9244 & 0.8029 & 0.6779 & 0.5693 \\
\hline & & 3 & 18 & 0.0170 & 0.9197 & 0.8185 & 0.6093 & 0.4417 & 0.3226 \\
\hline & \multirow[t]{5}{*}{15} & \multirow[t]{3}{*}{1} & 8 & 0.5770 & 0.0669 & 0.5378 & 0.8251 & 0.9087 & 0.9141 \\
\hline & & & 12 & 0.5770 & 0.2786 & 0.8772 & 0.9712 & 0.9625 & 0.9347 \\
\hline & & & 18 & 0.5770 & 0.7017 & 0.9913 & 0.9865 & 0.9648 & 0.9351 \\
\hline & & 2 & 18 & 0.2238 & 0.7014 & 0.9799 & 0.9364 & 0.8593 & 0.7728 \\
\hline & & 3 & 18 & 0.0661 & 0.7005 & 0.9495 & 0.8361 & 0.6904 & 0.5565 \\
\hline & \multirow[t]{5}{*}{20} & \multirow[t]{3}{*}{1} & 8 & 0.6912 & 0.0002 & 0.0262 & 0.1338 & 0.2850 & 0.4251 \\
\hline & & & 12 & 0.7692 & 0.0748 & 0.6772 & 0.9324 & 0.9758 & 0.9711 \\
\hline & & & 18 & 0.7692 & 0.3714 & 0.9613 & 0.9952 & 0.9884 & 0.9739 \\
\hline & & 2 & 18 & 0.4451 & 0.3714 & 0.9593 & 0.9794 & 0.9423 & 0.8870 \\
\hline & & 3 & 18 & 0.2042 & 0.5000 & 0.9752 & 0.9390 & 0.8455 & 0.7348 \\
\hline
\end{tabular}

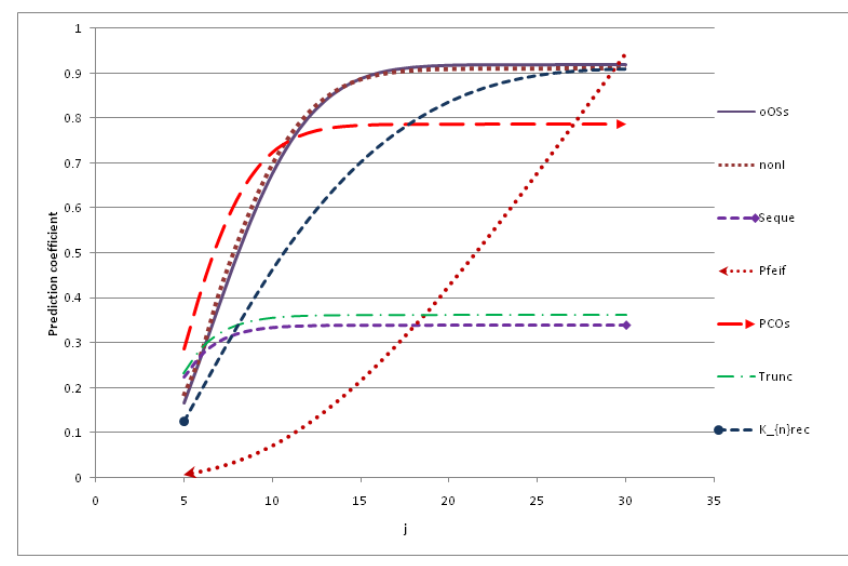

Figure 1: The coverage probabilities of the event $X_{3: 30} \leq Y_{5,20, \widetilde{m}^{*}, k^{*}} \leq X_{j: 30}$ under table 3 assumptions.

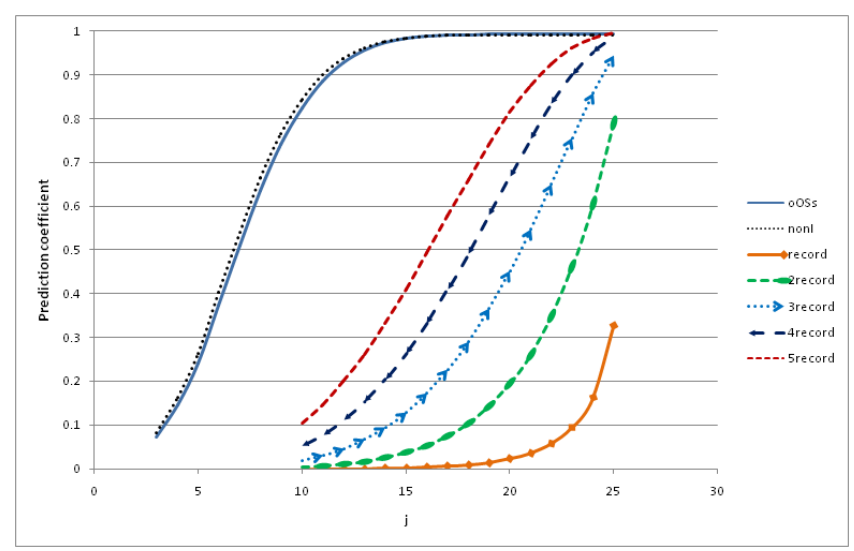

Figure 2: The coverage probabilities of the event $X_{1: 25: 30} \leq Y_{5,20, \widetilde{m}^{*}, k^{*}} \leq$ $X_{j: 25: 30}$ under table 4(a) assumptions. 


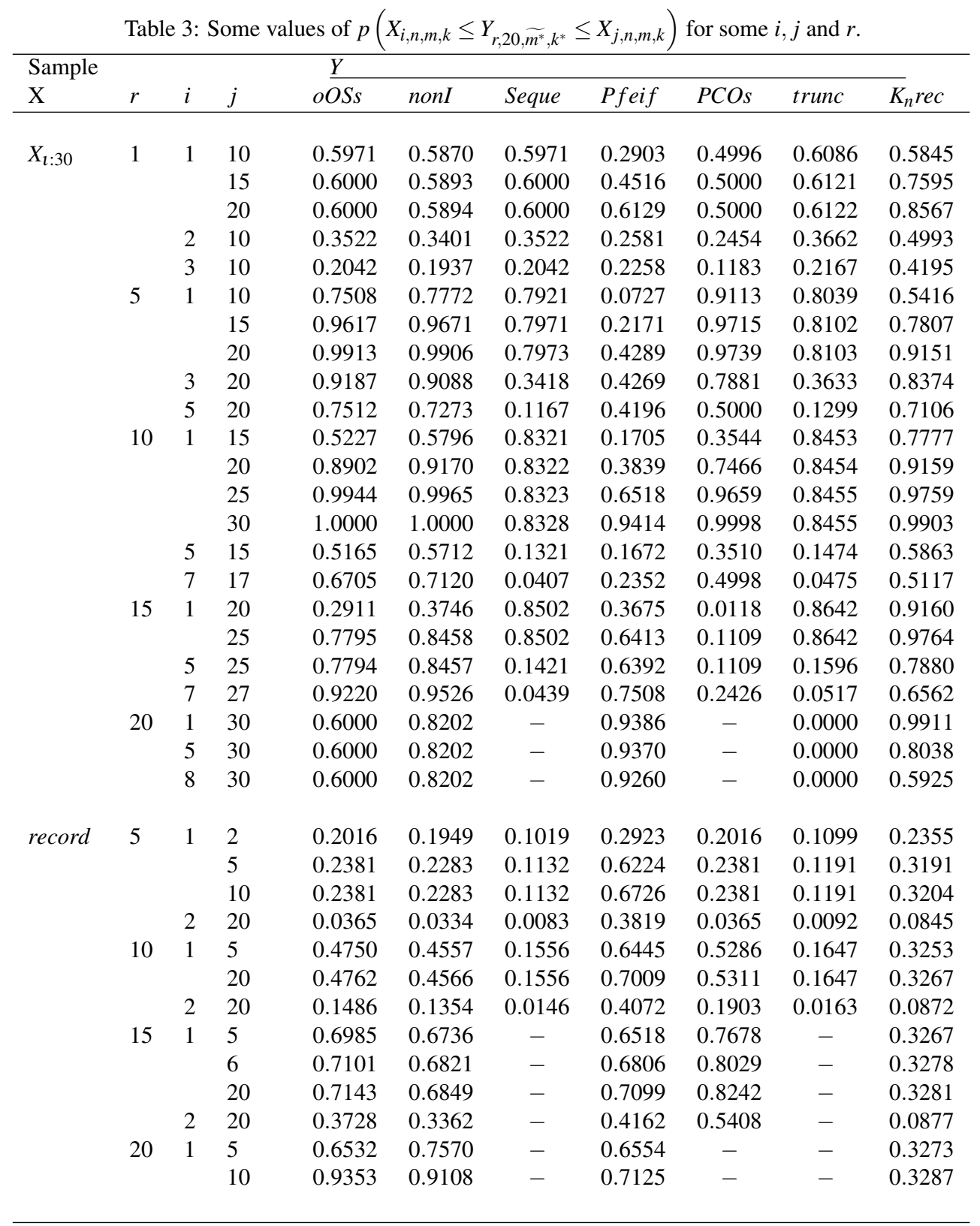

Proof. Based on lemma1, we can write

$$
\begin{aligned}
\phi_{1}\left(i, m ; r, m^{*}\right) & =P\left(X_{i, n, m, k}>Y_{r, n^{*}, m^{*}, k^{*}}\right) \\
& =\int_{-\infty}^{\infty} P\left(X_{i, n, m, k}>y\right) f_{Y_{r, n^{*}, m^{*}, k^{*}}}(y) d y .
\end{aligned}
$$

Upon substituting the survival function (3) and constructing pdf of $Y_{r, n^{*}, m^{*}, k^{*}}$ from (1), we can express

$$
\phi_{1}\left(i, m ; r, m^{*}\right)=\sum_{v=0}^{i-1} C_{v}(i ; r) I_{V}(i),
$$

such that

$$
\begin{aligned}
I_{v}(i) & =\int_{-\infty}^{\infty} \bar{F}^{\gamma_{i}+\gamma_{r}^{*}-1}(y) g_{m}^{v}(F(y)) g_{m^{*}}^{r-1}(F(y)) f(y) d y \\
& =\int_{0}^{1} y^{\gamma_{i}+\gamma_{r}^{*}-1} g_{m}^{v}(1-y) g_{m^{*}}^{r-1}(1-y) d y .
\end{aligned}
$$

The integration (11) is given for all $m, m^{*} \in \mathbf{R}$. Thus, based on assumptions of case $\mathrm{I}, I_{V}(i)$ is given by:

$$
\begin{cases}\int_{0}^{1} y^{\gamma_{i}+\gamma_{r}^{*}-1}\left(\frac{1-y^{m+1}}{m+1}\right)^{v}\left(\frac{1-y^{m^{*}+1}}{m^{*}+1}\right)^{r-1} d y, & m \neq-1 ; m^{*} \neq-1, \\ \int_{0}^{1} y^{\gamma_{i}+k^{*}-1}\left(\frac{1-y^{m+1}}{m+1}\right)^{v}(-\ln y)^{r-1} d y, & m \neq-1 ; m^{*}=-1, \\ \int_{0}^{1} y^{k+\gamma_{r}^{*}-1}(-\ln y)^{v}\left(\frac{1-y^{m^{*}+1}}{m^{*}+1}\right)^{r-1} d y, & m=-1 ; m^{*} \neq-1, \\ \int_{0}^{1} y^{k+k^{*}-1}(-\ln y)^{v+r-1} d y & \\ =\int_{0}^{\infty} z^{v+r-1} e^{-\left(k+k^{*}\right) z}=\frac{(v+r-1) !}{\left(k+k^{*}\right)^{v+r}}, & m=-1 ; m^{*}=-1 .\end{cases}
$$

Now, using the binomial expansion on some brackets and solving (12) as the last one, we obtain the required result. Using lemma 1 and under assumptions of theorem 1 , the coverage probability of the event $X_{i, n, m, k} \leq Y_{r, n^{*}, m^{*}, k^{*}} \leq X_{j, n, m, k}$, is given by:

$p\left(X_{i, n, m, k} \leq Y_{r, n^{*}, m^{*}, k^{*}} \leq X_{j, n, m, k}\right)=\phi_{1}\left(j, m ; r, m^{*}\right)-\phi_{1}\left(i, m ; r, m^{*}\right)$, 


\begin{tabular}{|c|c|c|c|c|c|c|c|c|c|c|}
\hline \multirow[b]{2}{*}{$X$} & \multirow[b]{2}{*}{$r$} & \multirow[b]{2}{*}{$i$} & \multirow[b]{2}{*}{$j$} & \multicolumn{3}{|l|}{$\underline{Y}$} & \multirow[b]{2}{*}{2 record } & \multirow[b]{2}{*}{3 record } & \multirow[b]{2}{*}{ 4record } & \multirow[b]{2}{*}{5 record } \\
\hline & & & & $o O S s$ & nonI & record & & & & \\
\hline \multirow{15}{*}{ PCOs } & \multirow[t]{2}{*}{1} & \multirow[t]{2}{*}{1} & 10 & 0.5984 & 0.5881 & 0.3610 & 0.5834 & 0.7233 & 0.8132 & 0.8719 \\
\hline & & & 20 & 0.6000 & 0.5894 & 0.7604 & 0.9357 & 0.9810 & 0.9939 & 0.9979 \\
\hline & \multirow[t]{2}{*}{3} & 1 & 10 & 0.9069 & 0.9074 & 0.0139 & 0.0738 & 0.1699 & 0.2814 & 0.3930 \\
\hline & & 2 & 10 & 0.7955 & 0.7885 & 0.0139 & 0.0738 & 0.1699 & 0.2814 & 0.3930 \\
\hline & \multirow[t]{3}{*}{5} & 1 & 10 & 0.8234 & 0.8443 & 0.0002 & 0.0045 & 0.0201 & 0.0535 & 0.1034 \\
\hline & & & 20 & 0.9926 & 0.9914 & 0.0237 & 0.1957 & 0.4522 & 0.6694 & 0.8135 \\
\hline & & 3 & 20 & 0.9200 & 0.9096 & 0.0237 & 0.1957 & 0.4522 & 0.6694 & 0.8135 \\
\hline & \multirow[t]{2}{*}{7} & 1 & 25 & 0.9992 & 0.9991 & 0.1209 & 0.5634 & 0.8411 & 0.9477 & 0.9831 \\
\hline & & 7 & 25 & 0.7632 & 0.7328 & 0.1209 & 0.5634 & 0.8411 & 0.9477 & 0.9831 \\
\hline & \multirow[t]{2}{*}{11} & \multirow[t]{2}{*}{8} & 23 & 0.9578 & 0.9463 & 0.0002 & 0.0184 & 0.1251 & 0.3244 & 0.5393 \\
\hline & & & 25 & 0.9603 & 0.9477 & 0.0105 & 0.1930 & 0.5115 & 0.7549 & 0.8883 \\
\hline & \multirow[t]{2}{*}{15} & \multirow[t]{2}{*}{1} & 24 & 0.9740 & 0.9854 & 0.0000 & 0.0055 & 0.0616 & 0.2094 & 0.4099 \\
\hline & & & 25 & 0.9946 & 0.9973 & 0.0007 & 0.0480 & 0.2319 & 0.4809 & 0.6906 \\
\hline & \multirow[t]{2}{*}{19} & \multirow[t]{2}{*}{1} & 24 & 0.5700 & 0.7320 & 0.0000 & 0.0004 & 0.0114 & 0.0653 & 0.1823 \\
\hline & & & 25 & 0.7995 & 0.8992 & 0.0000 & 0.0103 & 0.0877 & 0.2560 & 0.4605 \\
\hline \multirow[t]{11}{*}{ Trunk } & \multirow[t]{3}{*}{5} & 3 & 10 & 0.6604 & 0.6369 & 0.1243 & 0.4907 & 0.7492 & 0.8798 & 0.9413 \\
\hline & & 4 & 10 & 0.3702 & 0.3453 & 0.1244 & 0.4907 & 0.7492 & 0.8798 & 0.9413 \\
\hline & & & 11 & 0.3703 & 0.3453 & 0.2068 & 0.6387 & 0.8585 & 0.9443 & 0.9770 \\
\hline & \multirow[t]{4}{*}{9} & 2 & 10 & 0.9908 & 0.9900 & 0.0050 & 0.1054 & 0.3256 & 0.5475 & 0.7129 \\
\hline & & 4 & 10 & 0.7850 & 0.7586 & 0.0050 & 0.1054 & 0.3256 & 0.5475 & 0.7129 \\
\hline & & & 12 & 0.7880 & 0.7608 & 0.0335 & 0.3319 & 0.6570 & 0.8417 & 0.9282 \\
\hline & & 6 & 14 & 0.2972 & 0.2646 & 0.1328 & 0.6375 & 0.8897 & 0.9669 & 0.9885 \\
\hline & \multirow[t]{2}{*}{13} & 2 & 15 & 0.9998 & 0.9997 & 0.0419 & 0.4613 & 0.8058 & 0.9380 & 0.9801 \\
\hline & & 7 & 15 & 0.4443 & 0.3911 & 0.0419 & 0.4613 & 0.8058 & 0.9362 & 0.9741 \\
\hline & \multirow[t]{2}{*}{19} & 3 & 18 & 0.9993 & 0.9999 & 0.0455 & 0.5649 & 0.8912 & 0.9761 & 0.9946 \\
\hline & & & 19 & 0.9998 & 0.9999 & 0.0999 & 0.7206 & 0.9515 & 0.9920 & 0.9986 \\
\hline
\end{tabular}

where $\phi_{1}$ is given in (8). Thus, we have a prediction interval $\left(X_{i, n, m, k}, X_{j, n, m, k}\right), 1 \leq i<j \leq n$, for $Y_{r, n^{*}, m^{*}, k^{*}}\left(1 \leq r \leq n^{*}\right)$, whose prediction coefficient given by (13), is free of $F$.

\section{Some special cases of $\phi_{1}$ :}

We have the following simpler expressions for some special cases one-sided prediction coefficient $\phi_{1}$ that given by (8), and the corresponding two-sided prediction coefficient that given by (13).

- Distribution-free upper pound PIs for $Y_{r: n^{*}}$ from a future Y-sample of the form $\left(-\infty, X_{i: n}\right)$, can be obtained as a special case from $\phi_{1}\left(i, m ; r, m^{*}\right)$ by setting $m=m^{*}=0, k=k^{*}=1, \gamma_{i}=n-i+1,1 \leq i \leq n$ and $\gamma_{r}^{*}=n^{*}-r+1,1 \leq r \leq n^{*}$, and has the following form

$p\left(X_{i: n} \geq Y_{r: n^{*}}\right)=\phi_{1}(i, 0 ; r, 0)=\sum_{v=0}^{i-1} r\left(\begin{array}{c}n-i+v \\ v\end{array}\right)\left(\begin{array}{c}n^{*} \\ r\end{array}\right) \sum_{\lambda=0}^{v}(-1)^{\lambda}\left(\begin{array}{c}v \\ \lambda\end{array}\right) B\left(r, n-i+n^{*}-r+2\right)$.

Distribution-free two sided PIs for $Y_{r: n^{*}}$ of the form $\left(X_{i: n}, X_{j: n}\right)$ which was discussed by Mohie El-Din et al [1] appear here as a special case, such that

$p\left(X_{i: n} \leq Y_{r: n^{*}} \leq X_{j: n}\right)=P\left(X_{j: n} \geq Y_{r: n^{*}}\right)-P\left(X_{i: n} \geq Y_{r: n^{*}}\right)$.

Similarly:

- Prediction interval of future record based on oOSs which was discussed by Ahmadi and Balakrishnan [5], can be given with Lemma1 by:

$p\left(X_{i: n} \geq U_{r}\right)=\phi_{1}(i, 0 ; r,-1)=\sum_{v=0}^{i-1}\left(\begin{array}{c}n-i+v \\ v\end{array}\right) \sum_{\lambda=0}^{v} \frac{(-1)^{\lambda}\left(\begin{array}{c}v \\ \lambda\end{array}\right)}{(n-i+\lambda+2)^{r}}$.

- Prediction interval of future oOSs based on record which was discussed by Ahmadi and Balakrishnan [5], is given by:

$p\left(U_{i} \leq Y_{r: n^{*}} \leq U_{j}\right)=\phi_{1}(j,-1 ; r, 0)-\phi_{1}(i,-1 ; r, 0)=\sum_{v=i}^{j-1} r\left(\begin{array}{c}n^{*} \\ r\end{array}\right) \sum_{\lambda=0}^{r-1} \frac{(-1)^{\lambda}\left(\begin{array}{c}r-1 \\ \lambda\end{array}\right)}{\left(n^{*}-r+\lambda+2\right)^{v+1}}$.

- Prediction interval of future record based on record also, which was discussed by Raqab and Balakrishnan [8], is given by:

$p\left(U_{i} \leq U_{r}^{*} \leq U_{j}\right)=\phi_{1}(j,-1 ; r,-1)-\phi_{1}(i,-1 ; r,-1)=\sum_{v=i}^{j-1}\left(\begin{array}{c}v+r-1 \\ v\end{array}\right) \frac{1}{2^{v+r}}$ 
Table 4(b): Some values of $p\left(X_{i, 30, \widetilde{m}, k} \leq Y_{r, 20, \widetilde{m}^{*}, k^{*}} \leq X_{j, 30, \widetilde{m}, k}\right)$ based on Seque and Pfief, for some $i, j$ and $r$.

\begin{tabular}{|c|c|c|c|c|c|c|c|c|c|c|}
\hline \multirow[b]{2}{*}{$X$} & \multirow[b]{2}{*}{$r$} & \multicolumn{5}{|c|}{$\underline{Y}$} & \multirow[b]{2}{*}{2 record } & \multirow[b]{2}{*}{3 record } & \multirow[b]{2}{*}{ 4record } & \multirow[b]{2}{*}{5 record } \\
\hline & & $i$ & $j$ & $Y_{r: 20}$ & nonI & record & & & & \\
\hline \multirow[t]{17}{*}{ Seque } & 1 & 1 & 5 & 0.7205 & 0.7151 & 0.2335 & 0.4027 & 0.5278 & 0.7220 & 0.6940 \\
\hline & & & 7 & 0.7478 & 0.7398 & 0.4027 & 0.6271 & 0.7585 & 0.8387 & 0.8894 \\
\hline & & & 9 & 0.7499 & 0.7416 & 0.5776 & 0.8036 & 0.9016 & 0.9477 & 0.9708 \\
\hline & & 2 & 5 & 0.4143 & 0.4045 & 0.2335 & 0.4027 & 0.5279 & 0.6221 & 0.6941 \\
\hline & & & 7 & 0.4417 & 0.4292 & 0.4027 & 0.6271 & 0.7585 & 0.8387 & 0.8894 \\
\hline & 2 & 1 & 7 & 0.9280 & 0.9255 & 0.1090 & 0.2902 & 0.4555 & 0.5872 & 0.6873 \\
\hline & & & 9 & 0.9392 & 0.9351 & 0.2356 & 0.5186 & 0.7071 & 0.8211 & 0.8889 \\
\hline & & & 11 & 0.9398 & 0.9356 & 0.4066 & 0.7315 & 0.8791 & 0.9435 & 0.9723 \\
\hline & & 3 & 11 & 0.4765 & 0.4584 & 0.4066 & 0.7315 & 0.8791 & 0.9435 & 0.9723 \\
\hline & 4 & 1 & 9 & 0.9883 & 0.9895 & 0.0218 & 0.1358 & 0.3038 & 0.4687 & 0.6058 \\
\hline & & & 13 & 0.9969 & 0.9964 & 0.1786 & 0.5654 & 0.8027 & 0.9126 & 0.9606 \\
\hline & & 3 & 13 & 0.8294 & 0.8144 & 0.0721 & 0.3241 & 0.5720 & 0.7432 & 0.8481 \\
\hline & 11 & 5 & 16 & 0.9713 & 0.9632 & 0.0054 & 0.1521 & 0.4603 & 0.7146 & 0.8612 \\
\hline & & & 19 & 0.9716 & 0.9634 & 0.0589 & 0.5197 & 0.8466 & 0.9570 & 0.9880 \\
\hline & 16 & 8 & 20 & 0.9491 & 0.9252 & 0.0093 & 0.2879 & 0.6933 & 0.8961 & 0.9672 \\
\hline & & 10 & 22 & 0.7381 & 0.6668 & 0.0550 & 0.5910 & 0.9048 & 0.9809 & 0.9961 \\
\hline & 20 & 12 & 22 & 0.9159 & 0.8409 & 0.0102 & 0.3469 & 0.7688 & 0.9364 & 0.9799 \\
\hline \multirow[t]{17}{*}{ Pfeif } & 5 & 1 & 10 & 0.2381 & 0.2283 & 0.2002 & 0.6024 & 0.8250 & 0.9228 & 0.9647 \\
\hline & & & 15 & 0.2381 & 0.2283 & 0.2558 & 0.6968 & 0.8939 & 0.9629 & 0.9865 \\
\hline & & 2 & 15 & 0.0649 & 0.0598 & 0.2558 & 0.6968 & 0.8939 & 0.9629 & 0.9865 \\
\hline & 7 & 1 & 10 & 0.3331 & 0.3194 & 0.0629 & 0.3633 & 0.6435 & 0.8121 & 0.9015 \\
\hline & & 5 & 10 & 0.0084 & 0.0069 & 0.0629 & 0.3633 & 0.6435 & 0.8121 & 0.9015 \\
\hline & 9 & 1 & 10 & 0.4271 & 0.4099 & 0.0176 & 0.1956 & 0.4576 & 0.6678 & 0.8039 \\
\hline & & 7 & 11 & 0.0064 & 0.0050 & 0.0192 & 0.2096 & 0.4825 & 0.6940 & 0.8260 \\
\hline & 11 & 1 & 11 & 0.5196 & 0.4994 & 0.0051 & 0.1058 & 0.3235 & 0.5443 & 0.7111 \\
\hline & & 5 & 17 & 0.0560 & 0.0463 & 0.0076 & 0.1496 & 0.4248 & 0.6665 & 0.8214 \\
\hline & 13 & 1 & 20 & 0.6174 & 0.5927 & 0.0023 & 0.0851 & 0.3154 & 0.5682 & 0.7548 \\
\hline & & & 30 & 0.6188 & 0.5935 & 0.0034 & 0.1181 & 0.4052 & 0.6775 & 0.8466 \\
\hline & 15 & 5 & 20 & 0.2088 & 0.1750 & 0.0060 & 0.0408 & 0.2025 & 0.4327 & 0.6393 \\
\hline & & & 25 & 0.2141 & 0.1782 & 0.0007 & 0.0498 & 0.2388 & 0.4916 & 0.7014 \\
\hline & 17 & 1 & 30 & 0.7885 & 0.7668 & 0.0002 & 0.0277 & 0.1729 & 0.4111 & 0.6370 \\
\hline & & 8 & 30 & 0.2156 & 0.1674 & 0.0002 & 0.0277 & 0.1729 & 0.4111 & 0.6370 \\
\hline & 19 & 1 & 30 & 0.7497 & 0.7979 & 0.0001 & 0.0128 & 0.1061 & 0.3003 & 0.5223 \\
\hline & & 8 & 30 & 0.3475 & 0.3060 & 0.0001 & 0.0128 & 0.1061 & 0.3003 & 0.5223 \\
\hline
\end{tabular}

- Prediction interval of future $g O S s$ case I based on $o O S s$, is given by:

$p\left(X_{i: n} \geq Y_{r, n^{*}, m^{*}, k^{*}}\right)=\sum_{v=0}^{i-1} \frac{\left(\begin{array}{c}n-i+v \\ v\end{array}\right) c_{r-1}}{(r-1) !} \sum_{\lambda=0}^{v}(-1)^{\lambda}\left(\begin{array}{l}v \\ \lambda\end{array}\right)\left\{\begin{array}{cc}\frac{B\left(r, \frac{n-i+1+\gamma_{r}^{*}+\lambda}{m^{*}+1}\right)}{\left(m^{*}+1\right)^{r}}, & m^{*} \neq-1, \\ \frac{(r-1) !}{\left(n-i+1+k^{*}+\lambda\right)^{r}}, & m^{*}=-1 .\end{array}\right.$

- Prediction interval of future $g O S s$ case I based on record, is given by:

$p\left(U_{i} \geq Y_{r, n^{*}, m^{*}, k^{*}}\right)=\sum_{v=0}^{i-1} \frac{1}{(r-1) ! v !} \begin{cases}c_{r-1}^{*} \sum_{\eta=0}^{r-1} \frac{v ! b_{\eta}^{r-1}\left(m^{*}\right)}{\left(\gamma_{r}^{*}+\eta\left(m^{*}+1\right)+1\right)^{v+1}}, & m^{*} \neq-1, \\ \frac{(v+r-1) !\left(k^{*}\right)^{r}}{\left(k^{*}+1\right)^{v+r}}, & m^{*}=-1 .\end{cases}$

- Prediction interval of future $o O S s$ based on $g O S s$ case I, is given by:

$p\left(X_{i, n, m, k} \geq Y_{r: n^{*}}\right)=\sum_{v=0}^{i-1} \frac{n^{*} !}{\left(n^{*}-r\right) !(r-1) ! v !} \begin{cases}\frac{c_{i-1}}{c_{i-v-1}} \sum_{\lambda=0}^{v} b_{\lambda}^{v}(m) B\left(r, \gamma_{i}+n^{*}-r+1+\lambda(m+1)\right), & m \neq-1, \\ k^{v} \sum_{\eta=0}^{r-1} \frac{v !(-1)^{\eta}\left(\begin{array}{c}r-1 \\ \eta\end{array}\right)}{\left(k+n^{*}-r+1+\eta\right)^{v+1}}, & m=-1 .\end{cases}$

- Prediction interval of future record based on $g O S s$ case I, given by:

$p\left(X_{i, n, m, k} \geq U_{r}^{*}\right)=\sum_{v=0}^{i-1} \frac{1}{(r-1) ! v !} \begin{cases}\frac{c_{i-1}}{c_{i-v-1}} \sum_{\lambda=0}^{v} \frac{(r-1) ! b_{\lambda}^{v}(m)}{\left(\gamma_{i}+1+\lambda(m+1)\right)^{r}}, & m \neq-1, \\ \frac{(v+r-1) ! k^{v}}{(k+1)^{v+r}}, & m=-1 .\end{cases}$

Theorem2. Let $\left\{X_{i, n, m, k}, 1 \leq i \leq n\right\}$ under assumption $m_{1}=m_{2}=\ldots=m_{n-1}=m$, and $\left\{Y_{r, n^{*}, \tilde{m}^{*}, k^{*}}, 1 \leq r \leq n^{*}\right\}$ under assumption $\gamma_{i} \neq \gamma_{j}$, $i, j=1,2, \ldots, n^{*}-1$ and $i \neq j$, be two independent $g O S s$ from continuous cdf $F$. then $\left(-\infty, X_{i, n, m, k}\right), 1 \leq i \leq n$, is distribution-free one-sided 
Table 5: Some values of $p\left(X_{i, 30, \widetilde{m}, k} \leq Y_{r, 20, \widetilde{m}^{*}, k^{*}} \leq X_{j, 30, \widetilde{m}, k}\right)$ for some $i, j$ and $r$.

\begin{tabular}{|c|c|c|c|c|c|c|c|c|c|c|}
\hline \multirow[b]{2}{*}{$X$} & \multicolumn{6}{|c|}{$\underline{Y}$} & \multirow[b]{2}{*}{ Pfeif } & \multirow[b]{2}{*}{ PCOs } & \multirow[b]{2}{*}{ trunc } & \multirow[b]{2}{*}{$\overline{K_{n}} r e c$} \\
\hline & $r$ & $i$ & $j$ & $o O S S$ & nonI & Seque & & & & \\
\hline \multirow[t]{14}{*}{ Seque } & 1 & 1 & 10 & 0.6000 & 0.6012 & 0.6000 & 0.8347 & 0.5000 & 0.6122 & 0.9021 \\
\hline & & & 30 & 0.6000 & 0.6012 & 0.6000 & 0.9677 & 0.5000 & 0.6122 & 0.9091 \\
\hline & & 2 & 10 & 0.2522 & 0.2534 & 0.2522 & 0.7723 & 0.1629 & 0.2650 & 0.7462 \\
\hline & 5 & 1 & 10 & 0.9926 & 0.9927 & 0.7973 & 0.7803 & 0.9739 & 0.8103 & 0.9779 \\
\hline & & 2 & 10 & 0.9070 & 0.9081 & 0.3931 & 0.7771 & 0.7850 & 0.4169 & 0.8962 \\
\hline & & 3 & 10 & 0.6769 & 0.6795 & 0.1358 & 0.7598 & 0.4573 & 0.1379 & 0.7274 \\
\hline & 10 & 1 & 10 & 0.9921 & 0.9918 & 0.8323 & 0.7611 & 0.9740 & 0.8455 & 0.9806 \\
\hline & & 2 & 12 & 0.9713 & 0.9974 & 0.4346 & 0.9035 & 0.9966 & 0.4565 & 0.9150 \\
\hline & & 3 & 12 & 0.9710 & 0.9717 & 0.1523 & 0.8939 & 0.9812 & 0.1662 & 0.7501 \\
\hline & 15 & 4 & 20 & 0.9922 & 0.9986 & 0.0429 & 0.9612 & 0.9986 & 0.0490 & 0.5466 \\
\hline & & & 30 & 0.9922 & 0.9986 & 0.0429 & 0.9614 & 0.9998 & 0.0490 & 0.5466 \\
\hline & & 6 & 30 & 0.8554 & 0.8598 & 0.0016 & 0.7801 & 0.9917 & 0.0019 & 0.2075 \\
\hline & 20 & 1 & 15 & 0.8678 & 0.8437 & - & 0.9835 & 0.0000 & 0.0000 & 0.9913 \\
\hline & & 4 & 20 & 0.9979 & 0.9967 & - & 0.9643 & 0.0000 & 0.0000 & 0.5478 \\
\hline \multirow[t]{12}{*}{ Pfief } & 2 & 1 & 10 & 0.8235 & 0.8242 & 0.7013 & 0.1792 & 0.7435 & 0.7137 & 0.5860 \\
\hline & & & 15 & 0.8380 & 0.8388 & 0.7045 & 0.3542 & 0.7457 & 0.7177 & 0.7980 \\
\hline & & 2 & 15 & 0.6386 & 0.6402 & 0.4419 & 0.3464 & 0.4870 & 0.4600 & 0.7441 \\
\hline & 6 & 1 & 10 & 0.6470 & 0.6697 & 0.7951 & 0.0696 & 0.7781 & 0.8074 & 0.5565 \\
\hline & & 3 & 18 & 0.9488 & 0.8201 & 0.3383 & 0.3480 & 0.9099 & 0.3602 & 0.8058 \\
\hline & & 4 & 29 & 0.9139 & 0.9119 & 0.1984 & 0.9390 & 0.8349 & 0.2163 & 0.8467 \\
\hline & 12 & 1 & 18 & 0.5800 & 0.5623 & 0.8321 & 0.3089 & 0.2581 & 0.8458 & 0.8852 \\
\hline & & 3 & 18 & 0.5799 & 0.5502 & 0.3737 & 0.3085 & 0.2581 & 0.3988 & 0.8124 \\
\hline & & 6 & 24 & 0.9604 & 0.9204 & 0.0697 & 0.6289 & 0.6289 & 0.7679 & 0.7007 \\
\hline & 18 & 1 & 25 & 0.3914 & 0.3321 & - & 0.6870 & 0.0000 & 0.8711 & 0.9803 \\
\hline & & & 26 & 0.5265 & 0.3223 & - & 0.7487 & 0.0000 & 0.8711 & 0.9847 \\
\hline & & & 27 & 0.6755 & 0.3001 & - & 0.8111 & 0.0000 & 0.8711 & 0.9875 \\
\hline \multirow[t]{12}{*}{ PCOs } & 3 & 1 & 10 & 0.8757 & 0.8763 & 0.6447 & 0.1495 & 0.8757 & 0.4546 & 0.6177 \\
\hline & & & 20 & 0.8813 & 0.8819 & 0.6450 & 0.6433 & 0.8814 & 0.6549 & 0.9560 \\
\hline & & 2 & 20 & 0.6940 & 0.6953 & 0.3510 & 0.6409 & 0.6940 & 0.3628 & 0.9171 \\
\hline & 6 & 4 & 20 & 0.6920 & 0.6917 & 0.0981 & 0.0865 & 0.6982 & 0.1052 & 0.4708 \\
\hline & & 6 & 20 & 0.4708 & 0.4681 & 0.0186 & 0.5812 & 0.5000 & 0.0207 & 0.6755 \\
\hline & 12 & 3 & 15 & 0.9059 & 0.9001 & 0.2210 & 0.2564 & 0.8101 & 0.2328 & 0.7844 \\
\hline & & & 20 & 0.9958 & 0.9732 & 0.2210 & 0.5660 & 0.9913 & 0.2328 & 0.8958 \\
\hline & 16 & 1 & 16 & 0.7422 & 0.7222 & 0.7315 & 0.3009 & 0.5000 & 0.7423 & 0.8842 \\
\hline & & & 20 & 0.9746 & 0.9521 & 0.7315 & 0.5584 & 0.8958 & 0.7423 & 0.9640 \\
\hline & & 2 & 20 & 0.9745 & 0.9517 & 0.4330 & 0.5583 & 0.8958 & 0.4477 & 0.9391 \\
\hline & 18 & 2 & 20 & 0.9291 & 0.8880 & 0.4372 & 0.5556 & 0.7429 & 0.4522 & 0.9393 \\
\hline & & 8 & 22 & 0.9778 & 0.9231 & 0.0031 & 0.6799 & 0.9158 & 0.0037 & 0.5356 \\
\hline
\end{tabular}

PI for the future $Y_{r, n^{*}, \widetilde{m}^{*}, k^{*}}$, with the corresponding prediction coefficient $\phi_{2}\left(i, m ; r, \widetilde{m}^{*}\right)$, that does not depend on the sampling distribution $F$, and is given by:

$$
\sum_{v=0}^{i-1}(r-1) ! C_{v}(i ; r) \sum_{\mu=1}^{r} a_{\mu}^{*}(r) \begin{cases}\frac{B\left(v+1, \frac{\gamma_{i}+\gamma_{\mu}^{*}}{m+1}\right)}{(m+1)^{v+1}}, & m \neq-1, \\ \frac{v !}{\left(k+\gamma_{\mu}^{k}\right)^{v+1}}, & m=-1 .\end{cases}
$$

Proof. Under the assumption that $\left\{Y_{r, n^{*}, \widetilde{m}^{*}, k^{*}}, 1 \leq r \leq n^{*}\right\}$ are continuous r.v.'s, we can write

$$
\begin{aligned}
\phi_{2}\left(i, m ; r, \widetilde{m}^{*}\right) & =P\left(X_{i, n, m, k}>Y_{r, n^{*}, \widetilde{m}^{*}, k^{*}}\right) \\
& =\int_{-\infty}^{\infty} P\left(X_{i, n, m, k}>y\right) f_{Y_{r, n^{*}, \tilde{m}^{*}, k^{*}}}(y) d y .
\end{aligned}
$$

Using (3) and (4), $\phi_{2}$ can be written as

$$
\phi_{2}\left(i, m ; r, \widetilde{m}^{*}\right)=\sum_{v=0}^{i-1} \frac{c_{i-1} c_{r-1}^{*}}{v ! c_{i-v-1}} \sum_{\mu=1}^{r} a_{\mu}^{*}(r) J_{v, \mu}(i),
$$

such that

$$
J_{v, \mu}(i)=\int_{-\infty}^{\infty} \bar{F}^{\gamma_{i}+\gamma_{\mu}^{*}-1}(y) g_{m}^{v}(F(y)) f(y) d y .
$$


Thus, based on $g_{m}($.$) and (2), J_{v, \mu}(i)$ is given by:

$$
\begin{aligned}
& J_{V, \mu}(i)= \\
& \left\{\begin{array}{l}
\int_{-\infty}^{\infty} \bar{F}^{\gamma_{i}+\gamma_{\mu}^{*}-1}(y)\left(\frac{1-\bar{F}^{m+1}(y)}{m+1}\right)^{v} f(y) d y, \quad m \neq-1, \\
\int_{-\infty}^{\infty} \bar{F}^{k+\gamma_{\mu}^{*}-1}(y)(-\ln \bar{F}(y))^{v} f(y) d y, \quad m=-1 .
\end{array}\right.
\end{aligned}
$$

Using the following transformation $\bar{F}(y)=u$, we get

$$
J_{v, \mu}(i)= \begin{cases}\int_{0}^{1} u^{\gamma_{i}+\gamma_{\mu}^{*}-1}\left(\frac{1-u^{m+1}}{m+1}\right)^{v} d u, & m \neq-1, \\ \int_{0}^{1} u^{k+\gamma_{\mu}^{*}-1}(-\ln u)^{v} d u, & m=-1 .\end{cases}
$$

The prediction coefficient $\phi_{2}\left(i, m ; r, \widetilde{m}^{*}\right)$, given in (23) is obtained directly by solving (28).

\section{Some special cases of $\phi_{2}$ :}

- Prediction interval of future $o O S s$ based on also $o O S s$, is given by:

$$
p\left(X_{i: n} \geq Y_{r: n^{*}}\right)=\phi_{2}(i, 0 ; r, \widetilde{0})=\sum_{v=0}^{i-1} r !\left(\begin{array}{c}
n-i+v \\
v
\end{array}\right)\left(\begin{array}{c}
n^{*} \\
r
\end{array}\right) \sum_{\mu=1}^{r} a_{\mu}^{*}(r) B\left(v+1, n-i+n^{*}-\mu+2\right) \text {. }
$$

- Prediction interval of future $o O S s$ based on record, is given by:

$$
p\left(U_{i} \geq Y_{r: n^{*}}\right)=\phi_{2}(i,-1 ; r, \widetilde{0})=\sum_{v=0}^{i-1} r !\left(\begin{array}{c}
n^{*} \\
r
\end{array}\right) \sum_{\mu=1}^{r} \frac{a_{\mu}^{*}(r)}{\left(n^{*}-\mu+2\right)^{v+1}} .
$$

- Prediction interval of future gOSs case II based on oOSs which was discussed by Mohie El-Din and Emam [11], is given by:

$$
P\left(X_{i: n} \geq Y_{r, n^{*}, \widetilde{m}^{*}, k^{*}}\right)=\phi_{2}\left(i, 0 ; r, \widetilde{m}^{*}\right)=\sum_{v=0}^{i-1} \frac{\left(\begin{array}{c}
n-i+v \\
v
\end{array}\right) c_{r-1}^{*}}{(r-1) !} \sum_{\mu=1}^{r} a_{\mu}^{*}(r) B\left(v+1, n-i+1+\gamma_{\mu}^{*}\right) .
$$

- Prediction interval of future $o O S s$ based on $g O S s$ case I which was discussed in [11], is given by:

$$
P\left(X_{i, n, m, k}>Y_{r: n^{*}}\right)=\phi_{2}(i, m ; r, \widetilde{0})=\sum_{v=0}^{i-1} \frac{r\left(\begin{array}{c}
n^{*} \\
r
\end{array}\right)}{v !} \sum_{\mu=1}^{r} a_{\mu}^{*}(r) \begin{cases}\frac{c_{i-1} B\left(v+1, \frac{\gamma_{i}+n^{*}-\mu+1}{m+1}\right)}{c_{i-v-1}(m+1)^{v+1}}, & m \neq-1, \\
\frac{v ! k^{v}}{\left(k+n^{*}-\mu+1\right)^{v+1}}, & m=-1 .\end{cases}
$$

- Prediction interval of future gOSs case II based on record, is given by:

$$
P\left(U_{i} \geq Y_{r, n^{*}, \widetilde{m}^{*}, k^{*}}\right)=\phi_{2}\left(i,-1 ; r, \widetilde{m}^{*}\right)=\sum_{v=0}^{i-1} \frac{c_{r-1}^{*}}{(r-1) ! v !} \sum_{\mu=1}^{r} a_{\mu}^{*}(r) \frac{v !}{\left(\gamma_{\mu}^{*}+1\right)^{v+1}}
$$

Under the assumption of lemma $1,\left(X_{i, n, m, k}, X_{j, n, m, k}\right), 1 \leq i<j \leq n$, is a distribution-free PIs for $Y_{r, n^{*}, \widetilde{m}^{*}, k^{*}}\left(1 \leq r \leq n^{*}\right)$, whose coverage probability is free of the parent distribution $F$, given by:

$p\left(X_{i, n, m, k} \leq Y_{r, n^{*}, \widetilde{m}^{*}, k^{*}} \leq X_{j, n, m, k}\right)=\phi_{2}\left(j, m ; r, \widetilde{m}^{*}\right)-\phi_{2}\left(i, m ; r, \widetilde{m}^{*}\right)$,

where $\phi_{2}$ is given in (23).

Proof. By assumption that $\left\{Y_{r, n^{*}, \widetilde{m}^{*}, k^{*}}, 1 \leq r \leq n^{*}\right\}$ are continuous r.v.'s, we have

$$
\begin{aligned}
\phi_{3}\left(i, \tilde{m} ; r, m^{*}\right) & =P\left(X_{i, n, \widetilde{m}, k}>Y_{r, n^{*}, m^{*}, k^{*}}\right) \\
& =\int_{-\infty}^{\infty} P\left(X_{i, n, \widetilde{m}, k}>y\right) f_{Y_{r, n^{*}, m^{*}, k^{*}}}(y) d y
\end{aligned}
$$

By using (5) and constructing the pdf of $Y_{r, n^{*}, m^{*}, k^{*}}$ from (1), $\phi_{3}$ take the form

$$
\phi_{3}\left(i, \tilde{m} ; r, m^{*}\right)=C(i ; r) \sum_{v=1}^{i} \frac{a_{v}(i)}{\gamma_{v}} \zeta_{v}(i)
$$

where

$$
\zeta_{v}(i)= \begin{cases}\int_{-\infty}^{\infty}(\bar{F}(y))^{\gamma_{v}+\gamma_{r}^{*}-1}\left(\frac{1-\bar{F}^{m^{*}+1}(y)}{m^{*}+1}\right)^{r-1} f(y) d y, & m^{*} \neq-1 \\ \int_{-\infty}^{\infty}(\bar{F}(y))^{\gamma_{v}+k^{*}-1}(-\ln (\bar{F}(y)))^{r-1} f(y) d y, & m^{*}=-1\end{cases}
$$


Making the transformation $\bar{F}(y)=u$, we get

$$
\zeta_{v}(i)= \begin{cases}\int_{0}^{1} u^{\gamma_{v}+\gamma_{r}^{*}-1}\left(\frac{1-u^{m^{*}+1}}{m^{*}+1}\right)^{r-1} d u, & m^{*} \neq-1, \\ \int_{0}^{1} u^{\gamma_{v}+k^{*}-1}(-\ln (u))^{r-1} d u, & m^{*}=-1 .\end{cases}
$$

By solving the integrations (39), easily we obtain the required result.

\section{Some special cases of $\phi_{3}$ :}

- Prediction coefficient of future $o O S s$ based on also $o O S s$, is given by:

$\phi_{3}(i, \widetilde{0} ; r, 0)=r(i !)\left(\begin{array}{c}n \\ i\end{array}\right)\left(\begin{array}{c}n^{*} \\ r\end{array}\right) \sum_{v=1}^{i} \frac{(-1)^{i-v} B\left(r, n-i+n^{*}-r+2\right)}{(v-1) !(i-v) !(n-v+1)}$.

- Prediction coefficient of future record based on $o O S s$, is given by:

$\phi_{3}(i, \widetilde{0} ; r,-1)=\left(\begin{array}{l}n \\ i\end{array}\right) \sum_{v=1}^{i} \frac{(-1)^{i-v} v\left(\begin{array}{l}i \\ v\end{array}\right)}{(n-v+1)(n-v+2)^{r}}$.

- Prediction coefficient of future gOSs case I based on $o O S s$, is given by:

$\phi_{3}\left(i, \widetilde{0} ; r, m^{*}\right)=\frac{n !}{(n-i) !(r-1) !} \sum_{v=1}^{i} \frac{a_{v}(i)}{n-v+1} \begin{cases}\frac{B\left(r, \frac{n-v+1+\gamma_{r}^{*} c_{r-1}^{*}}{\left(m^{*}+1\right)^{r}}\right)}{m^{r}}, & m^{*} \neq-1, \\ \frac{(r-1) !}{\left(\frac{n-v+1}{k^{*}}+1\right)^{r}}, & m^{*}=-1 .\end{cases}$

- Prediction coefficient of future $o O S s$ based on $g O S s$ case II, is given by:

$\phi_{3}(i, \widetilde{m} ; r, 0)=r\left(\begin{array}{c}n^{*} \\ r\end{array}\right) c_{i-1} \sum_{v=1}^{i} \frac{a_{v}(i)}{\gamma_{v}} B\left(r, \gamma_{v}+n^{*}-r+1\right)$.

- Prediction coefficient of future record based on gOSs case II, is given by:

$\phi_{3}(i, \tilde{m} ; r,-1)=\frac{c_{i-1}}{(r-1) !} \sum_{v=1}^{i} \frac{a_{v}(i)}{\gamma_{v}} \frac{(r-1) !}{\left(\gamma_{v}+1\right)^{r}}$

Under assumptions of theorem 3 , then $\left(X_{i, n, \widetilde{m}, k}, X_{j, n, \widetilde{m}, k}\right), 1 \leq i<j \leq n$, is a distribution-free PIs for $Y_{r, n^{*}, m^{*}, k^{*}}\left(1 \leq r \leq n^{*}\right)$, whose coverage probability is free of the parent distribution $F$, is given by:

$p\left(X_{i, n, \widetilde{m}, k} \leq Y_{r, n^{*}, m^{*}, k^{*}} \leq X_{j, n, \widetilde{m}, k}\right)=\phi_{3}\left(j, \widetilde{m} ; r, m^{*}\right)-\phi_{3}\left(i, \widetilde{m} ; r, m^{*}\right)$. 
Theorem4. Let $\left\{X_{i, n, \tilde{m}, k}, 1 \leq i \leq n\right\}$ under assumption $\gamma_{i} \neq \gamma_{j}$, $i, j=1,2, \ldots, n-1$ and $i \neq j$ and $\left\{Y_{r, n^{*}, \widetilde{m}^{*}, k^{*}}, 1 \leq r \leq n^{*}\right\}$ under assumption $\gamma_{i}^{*} \neq \gamma_{j}^{*}, i, j=1,2, \ldots, n^{*}-1$ and $i \neq j$ be two independent $g O S s$ from continuous cdf $F$. then $\left(-\infty, X_{i, n, \widetilde{m}, k}\right), 1 \leq i \leq n$, is distribution-free one-sided PI for the future $Y_{r, n^{*}, \widetilde{m}^{*}, k^{*}}$, with the corresponding prediction coefficient $\phi_{4}(i, \mu ; r)$, that does not depend on the sampling distribution $F$, and is given by:

$$
\begin{aligned}
\phi_{4}\left(i, \widetilde{m} ; r, \widetilde{m}^{*}\right)=c_{i-1} c_{r-1}^{*} \sum_{v=1}^{i} \frac{a_{v}(i)}{\gamma_{v}} \sum_{\mu=1}^{r} \frac{a_{\mu}^{*}(r)}{\gamma_{v}+\gamma_{\mu}^{*}} . & (46) \\
\phi_{4}\left(i, \tilde{m} ; r, \tilde{m}^{*}\right) & =\int_{-\infty}^{\infty} c_{i-1} \sum_{v=1}^{i} \frac{a_{v}(i)}{\gamma_{v}}(1-F(y))^{\gamma_{v}} c_{r-1}^{*} f(y) \sum_{\mu=1}^{r} a_{\mu}^{*}(r)(1-F(y))^{\gamma_{\mu}^{*}-1} d y \\
& =c_{i-1} \sum_{v=1}^{i} \frac{a_{v}(i)}{\gamma_{v}} c_{r-1}^{*} \sum_{\mu=1}^{r} a_{\mu}^{*}(r) \int_{-\infty}^{\infty}(1-F(y))^{\gamma_{v}+\gamma_{\mu}^{*}-1} f(y) d y .
\end{aligned}
$$

Proof. Using assumptions, we found that

$$
\begin{aligned}
\phi_{4}\left(i, \widetilde{m} ; r, \widetilde{m}^{*}\right) & =P\left(X_{i, n, \widetilde{m}, k}>Y_{r, n^{*}, \widetilde{m}^{*}, k^{*}}\right) \\
& =\int_{-\infty}^{\infty} P\left(X_{i, n, \widetilde{m}, k}>y\right) f_{Y_{r, n^{*}, \widetilde{m}^{*}, k^{*}}}(y) d y .
\end{aligned}
$$

Using (4) and (5), $\phi_{4}$ has the following form
Using the transformation $\bar{F}(y)=u$, and solving the integration, easily we obtain the required result. Based on $\phi_{4}$ that given in (46) and under theorem 4 assumptions, then $\left(X_{i, n, \widetilde{m}, k}, X_{j, n, \widetilde{m}, k}\right), 1 \leq i<$ $j \leq n$, is a distribution-free PIs for $Y_{r, n^{*}, \widetilde{m}^{*}, k^{*}}\left(1 \leq r \leq n^{*}\right)$, whose coverage probability is free of the parent distribution $F$, given by:

$p\left(X_{i, n, \widetilde{m}, k} \leq Y_{r, n^{*}, \widetilde{m}^{*}, k^{*}} \leq X_{j, n, \widetilde{m}, k}\right)=\phi_{4}\left(j, \widetilde{m} ; r, \widetilde{m}^{*}\right)-\phi_{4}\left(i, \widetilde{m} ; r, \widetilde{m}^{*}\right)$,

\section{Numerical Results}

In section 3, distribution-free PIs for future gOSs based on also $g O S s$ is constructed. To illustrate the productive prediction coefficient for some choices of $i, j$ and $r$, and by using some different choices of $\gamma_{i}$ and $\gamma_{r}^{*}$ in table 1 to gaining the special schemes of the gOSs. Table 2 presents some values of the coverage probability $p\left(X_{i, 20, m, k} \leq Y_{r, n^{*}, m^{*}, k^{*}} \leq X_{j, 20, m, k}\right)$ of future, oOSs (by setting $\gamma_{r}^{*}=n^{*}-r+1$ ) with $n^{*}=25$ and Krecord (by setting $\gamma_{r}^{*}=K$ ) with $K=1,2,3,4$ and 5, based on oOSs and record consecutively, such that $p\left(X_{i, n, m, k} \leq Y_{r, n^{*}, m^{*}, k^{*}} \leq X_{j, n, m, k}\right)$ does not depend on the parent distribution $F$, given by (13). Some values of the coverage probability $p\left(X_{i, n, m, k} \leq Y_{r, 20, \widetilde{m^{*}, k^{*}}} \leq X_{j, n, m, k}\right)$ of future oOSs, nonI (by setting $\left.\gamma_{r}=\left(n^{*}+0.9\right)-r+1\right)$, Seque (using $\alpha_{r}=r^{2}$ ), Pfeif (by setting $\gamma_{r}=r^{2}$ ), PCOs (using $r_{1}=5, n_{1}=5$ ), Trunc (using $\alpha_{r}=r^{2}, k_{r}=n^{*}-r$ ) and $K_{n} r e c$ (using $\beta_{r}=r^{2}, k_{r}=r+2$ ), based on $o O S s$ with $n=30$ and record are presented respectively, in table 3 , with the coverage probability that given by (34).

Table 4(a) presents some values of $p\left(X_{i, 30, \widetilde{m}, k} \leq Y_{r, n^{*}, m^{*}, k^{*}} \leq X_{j, 30, \widetilde{m}, k}\right)$ of future oOSs and Krecord, $K=1,2,3,4$ and 5 based on PCOs (using $r_{1}=5, n_{1}=5$ ) and Trunc (using $\alpha_{r}=r^{-1}, k_{r}=n^{*}-r$ ), respectively. Under the same assumption, table 4(b) holds based on Seque (using $\alpha_{r}=2 r^{-1}$ ) and Pfeif (by setting $\gamma_{r}=r^{2}$ ), respectively. Table 5 presents some values of $p\left(X_{i, 30, \widetilde{m}, k} \leq Y_{r, 20, \widetilde{m}^{*}, k^{*}} \leq X_{j, 30, \widetilde{m}, k}\right)$ based on Seque (using $\alpha_{r}=r^{-1}$ ), Pfeif (by setting $\gamma_{r}=n-r$ ) and PCOs (using $r_{1}=5, n_{1}=5$ ), respectively, the prediction coefficient of future oOSs, nonI (using $\left.\alpha_{r}=\left(n^{*}-0.1\right)-r+1\right)$ ), Seque (using $\alpha_{r}=r^{2}$ ), Pfeif (by setting $\gamma_{r}=r^{2}$ ), PCOs (using $r_{1}=5, n_{1}=5$ ), Trunc (using $\alpha_{r}=r^{2}, k_{r}=n^{*}-r$ ) and $K_{n} r e c$ (using $\beta_{r}=r^{2}, k_{r}=r+2$ ), respectively.
Under table 3 assumptions, figure 1 plots $p\left(X_{3,30,0,1} \leq Y_{5,20, \widetilde{m}^{*}, k^{*}} \leq X_{j, 30,0,1}\right)$, which presents the coverage probability of future $5^{\text {th }} \mathrm{gOSs}$ case II based on oOSs. Therefore, $\left(X_{3: 30}, X_{j: 30}\right), 1 \leq j \leq 30$, is a distribution-free PIs for $Y_{5,20, \widetilde{m}^{*}, k^{*}}$. Under table 4(a) assumptions, figure 2 plots the coverage probability of future $5^{\text {th }}$ gOSs case I based on PCOs. Then, $\left(X_{1: 25: 30}, X_{j: 25: 30}\right)$, $1 \leq j \leq 30$, is a distribution-free PIs for $Y_{5,20, m^{*}, k^{*}}$.

\section{Conclusions}

The prediction of unobserved statistics arises naturally in several real life situations. In This paper, nonparametric PIs for some statistics in a future unobserved $\mathrm{gOSs}$ based on $\mathrm{gOS}$ s from the same underlying distribution $F()$ are constructed. The proposed procedure can be extended to construct the outer and inner prediction intervals for future $g O S s$ based on $g O S s$. The following conclusions are noted here:

$\circ$ The prediction coefficient are decreasing with $i$ and increasing with $j$, as it was expected.

$\circ$ All prediction coefficients under the same assumptions are equivalent, for example

$\phi_{1}(i, 0 ; r ; 0)=\phi_{2}(i, 0 ; r ; \widetilde{0})=\phi_{3}(i, \widetilde{0} ; r ; 0)=\phi_{4}(i, \widetilde{0} ; r ; \widetilde{0})=p\left(X_{i: n} \geq Y_{r: n^{*}}\right)$.

○ The generality of our work enabled us to compare the values of different future sampling schemes at the same time, and choose the best one corresponding with the practical work.

- Under the same assumptions, the prediction coefficients of future Krecord are increasing with $K$.

\section{References}

[1] M. M. Mohie El-Din, M. S. Kotb, W. S. Emam, Prediction intervals for future order statistics from two independent sequences, AJTAS, 4 (2015) $33-40$.

[2] M. M. Mohie El-Din, M. S. Kotb, W. S. Emam, Prediction intervals for progressive Type-II right-censored order statistics, AJTAS, 4 (2015) $329-338$.

[3] J. Ahmadi, N. Balakrishnan, Confidence intervals for quantiles in terms of record range, Stat. Prob. Letters 68 (2004) 395 - 405.

[4] J. Ahmadi, N. Balakrishnan, Distribution-free confidence intervals for quantile intervals based on current records, Stat. Prob. Letters 75 (2005) $190-202$. 
[5] J. Ahmadi, N. Balakrishnan, Prediction of order statistics and record values from two independent sequences, Statistics 44 (2010) 417-430.

[6] J. Ahmadi, N. Balakrishnan, Distribution-free prediction intervals for order statistics based on record coverage, J. Korean Stat. Society 40 (2011) $181-192$.

[7] J. Ahmadi, S.M.T.K. MirMostafaee, N. Balakrishnan, Nonparametric prediction intervals for future record intervals based on order statistics, Stat. Prob. Letters 80 (2010) 1663 - 1672

[8] M. Z. Raqab, N. Balakrishnan, Prediction intervals for future records, Stat. Prob. Letters, 78 (2008) 1955 - 1963.

[9] M.Z. Raqab, Distribution-free prediction intervals for the future current record statistics, Springer 50 (2009) 429-439.

[10] D. Krewski, Distribution-free confidence intervals for quantile intervals, J. American Stat. Association, 71 (1976) $420-422$.

[11] M. M. Mohie El-Din, W. S. Emam, Prediction of generalized order statistics based on ordinary order statistics, BOMSR, 3 (2015) 205 215

[12] U. Kamps, A concept of generalized order statistics, Teubner, Stuttgart (1995).

[13] U. Kamps, E. Cramer, On distributions of generalized order statistics, Statistics 35 (2001) $269-280$. 